\title{
Erythropoietin Receptor Signaling Supports Retinal Function after Vascular Injury
}

Colin A. Bretz, Aaron B. Simmons, Eric Kunz, Aniket Ramshekar, Carson Kennedy, Ivan Cardenas, and M. Elizabeth Hartnett

From the John A. Moran Eye Center, University of Utah, Salt Lake City, Utah

Accepted for publication

November 19, 2019.

Address correspondence to $\mathrm{M}$. Elizabeth Hartnett, M.D., $65 \mathrm{~N}$ Mario Capecchi Dr., Salt Lake City, UT 84132. E-mail: me. hartnett@hsc.utah.edu.

\begin{abstract}
The investigation of erythropoietin (EPO) has expanded to include potential nonhematopoietic roles in neural and retinal diseases, including diabetic retinopathy. However, it remains unclear how EPO functions to support the neural retina. Transgenic mice with hypoactive EPO receptor (EPOR) signaling (hWtEPOR) were compared with littermate control mice (WT) to test the role of EPOR signaling under normal conditions and after vascular injury and regrowth into the retina. Although retinal function tested with OptoMotry and electroretinography was comparable to adult (8-week-old) littermate WT mice, hWtEPOR mice had thinner inner and outer plexiform layers and a greater number of amacrine cells. Injury and repair caused by the oxygen-induced retinopathy model reduced visual acuity thresholds, reduced electroretinography amplitudes, and thinned the outer plexiform and inner nuclear layers of both WT and hWtEPOR 8-week-old mice. In hWtEPOR compared with WT mice, scotopic a-wave amplitudes were reduced by injury, despite no change in outer nuclear layer thickness; and peripheral rod, but not cone number, was reduced. Scotopic b-waves were reduced in injured hWtEPOR mice compared with WT, and rod bipolar cell ectopic neurites were increased in both genotypes after injury, suggesting a potential reparative process to preserve connectivity and the b-wave. Normal EPOR signaling appeared important because ectopic neurites and b-waves were lower in the hWtEPOR than WT injured mice. (Am J Pathol 2020, 190: 630-641; https://doi.org/10.1016/j.ajpath.2019.11.009)
\end{abstract}

Evidence suggests that the hematopoietic hormone, erythropoietin (EPO), has tissue and/or neural protective properties. ${ }^{1-3}$ Thus, EPO, given systemically, is being tested in extreme prematurity to reduce neurocognitive deficits in later childhood and is being considered to protect against retinal damage in diabetes. ${ }^{4-7}$ Studies to date are either ongoing or have not reported definitive data. We and others have also found evidence of angiogenic properties of EPO. For example, EPO reduced high oxygen-induced loss of capillaries in a murine model of oxygen-induced retinopathy (OIR), ${ }^{8}$ but was involved in pathologic angiogenesis into the vitreous in other instances. ${ }^{9-13}$ However, less is known about the effects of EPO on vascular and tissue interactions in neural or tissue protection.

EPO triggers signaling through its receptor, EPOR, which can form a homodimer or has been proposed to form heterodimers with vascular endothelial growth factor receptor 2 to augment angiogenesis or the $\beta$ common receptor to enhance tissue protection. ${ }^{10,14,15}$ Herein, we addressed the hypothesis that signaling through EPOR would protect against retinal neural damage in development and after vascular loss and repair by using the mouse model of OIR in which high oxygen causes first loss of retinal capillaries, followed by vascular regrowth of the retina when animals are returned to ambient conditions. ${ }^{16}$ The vascular damage in the mouse OIR model occurs consistently in the central retina in the region surrounding the optic nerve and provides a comparison to the peripheral retina. This model may represent several retinovascular diseases, including retinal vein occlusions, inflammatory vasculitis, and diabetic retinopathy, in which there is blocked blood flow, followed by reperfusion or remodeling of the vasculature, as well as

Supported by the NIH grant EY014800; a Research to Prevent Blindness, Inc. unrestricted grant (John A. Moran Eye Center, Department of Ophthalmology and Visual Sciences, University of Utah); and National Eye Institute/NIH grants R01 EY015130 and R01 EY017011 (M.E.H.).

Disclosures: None declared. 
certain aspects of retinopathy of prematurity from high oxygen-induced attenuation.

To assess the effect of EPOR signaling on neural retinal function and structure, humanized knock-in mice in which a wild-type human EPOR gene (hWtEPOR) was knocked into the endogenous mouse EpoR locus, replacing the wild-type murine EpoR gene, were used. ${ }^{17}$ The expressed human EPOR is still able to bind endogenous mouse EPO, but homozygous hWtEPOR mice have hypoactive EPOR signaling compared with homozygous murine EpoR littermate controls, ${ }^{17,18}$ because of decreased stability of a transmembrane protein involved in receptor dimerization and the activation of downstream signaling. ${ }^{19}$

In our model, hypoactive EPOR signaling interferes with normal neural retinal structure without causing measured abnormalities in visual behavior experiments. Furthermore, induced vascular loss, followed by repair in mice with hypoactive EPOR signaling, led to reduced numbers of amacrine cells and rod bipolar cell ectopic neurites in association with reduced scotopic a-waves, b-waves, and oscillatory potential amplitudes compared with mice with normal EPOR signaling that sustained induced vascular loss followed by repair. Our findings support the idea that after retinovascular loss and repair, there is a repatterning of neurons within the retina to maintain connectivity of rod photoreceptors through rod bipolar cell ectopic neurites that extend into the outer nuclear layer and limit the loss of the b-wave, and that normal EPOR signaling is important in these processes.

\section{Materials and Methods}

Mice

All animal protocols were approved by the Institutional Animal Care and Use Committee and the Institutional Biosafety Committee of the University of Utah (Salt Lake City, UT), and animal procedures were conducted in accordance with the University of Utah Guide for the Care and Use of Laboratory Animals and the Association for Research in Vision and Ophthalmology Statement for the Use of Animals in Ophthalmic and Vision Research. For all studies, heterozygous hWtEPOR/mWtEpoR mice were bred to obtain litters that contained heterozygous hWtEPOR/ $\mathrm{mWtEpoR}$ mice, homozygous hWtEPOR/hWtEPOR mice (denoted hWtEPOR throughout), and homozygous $\mathrm{mWt} E$ $p o R / \mathrm{mWtEpoR}$ mice (denoted WT throughout). Only homozygous hWtEPOR and WT mice were used as experimental animals for analysis. The mouse colony is maintained on a $\mathrm{C} 57 \mathrm{Bl} / 6 \mathrm{~J}$ background, and backcrossed to C57B1/6J mice (Jackson Laboratory, Bar Harbor, ME) every five generations. The colony was monitored for $R d 1, R d 8$, and Gnat2. ${ }^{20}$ Male and female mice were used in all experiments.

\section{Oxygen-Induced Retinopathy Model}

To produce injury followed by repair, the mouse OIR model was used. ${ }^{21}$ On postnatal day 7 , litters and dams from heterozygous hWtEPOR/mWtEpoR breeding pairs were placed in an OxyCycler (Biospherix, Parish, NY) and exposed to $75 \%$ oxygen for 5 days. On postnatal day 12, litters were removed from the OxyCycler and returned to room air. Litters were weaned and genotyped at postnatal day 21. No animals exposed to oxygen treatment, dams, or heterozygous pups were used for additional breeding to avoid any potential effects of OIR.

\section{OKT Response}

As a measure of visual function, visual acuity thresholds were determined at 4,6 , and 8 weeks of age by observing the optomotor kinetic tracking (OKT) response to visual stimuli using the OptoMotry system (CerebralMechanics, Inc., Lethbridge, AB, Canada), as previously described. ${ }^{22}$ For scotopic analysis, mice were dark adapted overnight. The liquid-crystal display screens in the OptoMotry system were covered with ND16 neutral density filters, and mice were handled under dim red light throughout the OKT response behavioral experiments. Mice were placed onto an elevated platform within the OptoMotry box that displays a virtual rotating cylinder with white and black lines in a sine wave grating pattern. A trained, masked observer (A.B.S.) monitored the behavior of each mouse by a live camera feed and indicated to the software when the mouse detected the visual stimulus, as defined by a reproducible smooth tracking response of the head and/or neck in the direction of the rotating cylinder. To measure visual acuity, the grating frequency (cycles per degree) was increased by a simple staircase method while the rotation speed (12 degrees/second) and contrast (100\%) were held constant. ${ }^{22}$ The visual capabilities of each eye were determined independently on the basis of the rotation of the stimulus (clock-wise rotation: left eye; counter clock-wise rotation: right eye). ${ }^{23,24}$ Visual acuity threshold was defined as the highest grating frequency responded to by the mouse in both directions. The trained observer was masked to all treatment conditions throughout the study.

\section{ERG Data}

At 8 weeks of age, retinal function of injured compared with age-matched uninjured control mice was assessed using an LKC UTAS Visual Diagnostic System with the BigShot Ganzfeld ERG (LKC Technologies, Gaithersburg, MD) under scotopic and photopic conditions. For scotopic electroretinograms (ERGs), mice were fully dark adapted overnight, and all procedures were conducted under dim red-light illumination. During ERG recording, mice were anesthetized by i.p. injection of ketamine and xylazine and kept on a heating pad to maintain constant body 
temperature. Pupils were dilated with $1 \%$ tropicamide eye drops (Bausch and Lomb, Tampa, FL). Subdermal needle electrodes were inserted between the eyes on the forehead to serve as the reference electrode and at the base of the tail to serve as the ground electrode. For scotopic ERGs, responses to light stimuli were elicited at luminances ranging from -2 to $2 \mathrm{~cd} \mathrm{~s} \mathrm{~m}^{-2}$. For photopic ERGs, luminances ranged from 0.4 to $1.9 \mathrm{~cd} \mathrm{~s} \mathrm{~m}^{-2}$. Oscillatory potential (OP) amplitudes were measured at the maximum scotopic intensity. A-wave, b-wave, and OP amplitudes were determined using UTAS software version 1.4 from LKC Technologies. Individuals responsible for recording ERGs and extracting amplitudes were masked to all treatment conditions throughout the study.

\section{Tissue Harvest and Preparation}

At 8 weeks of age, animals were sacrificed in accordance with Institutional Animal Care and Use Committee protocols, and eyes were collected for flat-mount and cryosection analyses. Eyes were enucleated, placed into 4\% paraformaldehyde fixative for an hour at room temperature with part of the cornea removed, and then washed immediately in phosphate-buffered saline (PBS). The cornea, lens, iris, and vitreous were then removed. For retinal flat mounts, the retina was dissected away from the posterior portion of the eye containing the retinal pigment epithelium, choroid, and sclera. For cryosections, eyes were cryopreserved by equilibrating the tissue in a $30 \%$ sucrose solution and embedded in a 2:1 Tissue-Tek OCT Compound (Sakura, Torrance, CA) $/ 30 \%$ sucrose solution by freezing on dry ice. Only the middle third of the embedded eye was used. From this third, sections taken $12 \mu \mathrm{m}$ apart were placed onto charged microscope slides.

\section{Immunohistochemistry}

To stain cryosections, mounting medium was removed by incubating the slides in PBS at room temperature before blocking the sections in a $0.1 \%$ Triton block solution. Sections were incubated with primary antibodies diluted to the appropriate concentration with $0.1 \%$ Triton block solution overnight at $4^{\circ} \mathrm{C}$, washed three times in PBS at room temperature, then incubated with secondary antibodies diluted in $0.1 \%$ Triton block for 3 hours at room temperature. Slides were again washed three times in PBS and mounted with mounting medium containing DAPI.

Retinas dissected for flat mounts were blocked in $0.4 \%$ Triton block solution overnight at $4{ }^{\circ} \mathrm{C}$. Retinas were incubated in primary and secondary antibodies diluted to the appropriate concentration in $0.4 \%$ Triton block solution for 3 days at $4^{\circ} \mathrm{C}$. Washes were performed after each antibody incubation, three times in PBS for 30 minutes at room temperature, followed by overnight incubation in $0.4 \%$ Triton block solution at $4^{\circ} \mathrm{C}$.

\section{Antibodies}

The following antibodies were used to identify specific classifications of retinal neurons: cone photoreceptors ${ }^{25}$ : rabbit anti-cone arrestin (catalog number AB15282; Millipore Sigma, Burlington, MA; 1:500); rod bipolar cells ${ }^{26}$ : mouse anti-protein kinase $\mathrm{C}$ alpha ( $\mathrm{PKC} \alpha$; catalog number sc-8393; Santa Cruz Biotechnology, Dallas, TX; 1:100); amacrine cells ${ }^{25}$ : mouse anti-activator protein $2 \alpha$ (AP2 $\alpha$; catalog number 5E4; deposited to the Developmental Studies Hybridoma Bank by T. J. Williams; 1:50); and ganglion cells ${ }^{27}$ : rabbit anti-RNA-binding protein with multiple splicing (RBPMS; catalog number 1830-RBPMS; PhosphoSolutions, Aurora, CO; 1:200).

\section{Retinal Thickness Measurements}

Cryosections of retina containing the optic nerve head $(\mathrm{ONH})$ from the middle third of embedded eyes were stained with DAPI, imaged at $\times 10$ magnification on an inverted Olympus fluorescent microscope (Center Valley, PA), and stitched together into montages using MetaMorph software version 7.8.2.0 (Molecular Devices, LLC, San Jose, CA) (Figure 3A). Montaged images were imported into FIJI software version 1.0 (NIH, Bethesda, MD), and thickness measurements of retinal layers and total retinal thickness were measured from four consecutive sections of a single eye per mouse. Trained scorers measured sections at $200,400,600,800$, and $1000 \mu \mathrm{m}$ from the ONH in both directions, taking care to ensure thickness measurements were done perpendicular to the outer extent of the outer nuclear layer (ONL). Retinal layers measured included the following: ONL, outer plexiform layer (OPL), inner nuclear layer (INL), inner plexiform layer (IPL), ganglion cell layer (GCL), and total retinal thickness. The extent of the cell body-containing layers (ONL, INL, and GCL) was assessed by DAPI staining for each respective layer. The OPL thickness was defined as the unstained area between the DAPI staining of the ONL and INL, and the IPL thickness as the unstained area between the INL and GCL. Total retinal thickness included thickness of the GCL to the ONL. One eye from at least six mice per treatment group was analyzed; and for each eye, four sections were measured, yielding eight measurements per eye at each distance from the ONH. Trained scorers were masked to all treatment conditions throughout the study. For analyses, the hypothesis was tested at either the $200 \mu \mathrm{m}$ location for central retina or the $1000 \mu \mathrm{m}$ location for peripheral retina.

\section{Individual Cell Type Counts}

Cryosections of retina containing the $\mathrm{ONH}$ were stained with cell-specific markers (Antibodies) and DAPI. Images of rod photoreceptors, cone photoreceptors, rod bipolar cells, and amacrine cells at central and peripheral locations relative to the $\mathrm{ONH}$ were captured at $\times 40$ magnification using 
an inverted scanning laser confocal microscope (Olympus). The central and peripheral locations on either side of the $\mathrm{ONH}$ were imaged from a single retina/mouse. Images were imported into FIJI software and cell nuclei double labeled for a specific marker and DAPI were manually counted by trained scorers using the multipoint tool. ${ }^{28,29}$ For cone photoreceptors, rod bipolar cells, and amacrine cells, the number of nuclei were counted per image and normalized per length of cell-specific layer. For rod photoreceptors, four columns of nuclei in the ONL were counted per image, excluding those stained by cone arrestin. For cell-specific counts in sections, one eye from at least four mice was analyzed per treatment condition.

The density of retinal ganglion cells was determined in retinal flat mounts. Flat mounts were stained with RBPMS (Antibodies) and imaged as above with the exception that four images from each central and peripheral region were captured from each retina. Ganglion cells per field were manually quantified using the multipoint tool in FIJI. For ganglion cell counts, one retina, yielding four central and four peripheral images, from at least three mice was analyzed for each treatment condition. Trained scorers were masked to all treatment conditions throughout the study.

\section{Statistical Analysis}

STATA14 software (StataCorp, College Station, TX) was used for statistical analyses. Analyses were conducted using a multilevel mixed-effects linear regression model to account for clustering of animals within litters in the OptoMotry and ERG studies and clustering of images from individual animals in the retinal thickness studies. Individual comparisons were made between uninjured WT and uninjured hWtEPOR mice, uninjured WT and injured WT mice, uninjured $\mathrm{hWt} E P O R$ mice and injured $\mathrm{hWt} E P O R$, and injured WT and injured hWtEPOR mice. For ERG and retinal thickness studies, a primary-secondary approach was used; and the hypotheses were tested at the highest luminance for ERGs and at the most central $(200 \mu \mathrm{m})$ or most peripheral $(1000 \mu \mathrm{m})$ distance for retinal thickness. Additional individual comparisons are reported descriptively. $P<0.05$ was considered statistically significant.

\section{Results}

Effect of Reduced EPOR Signaling on Retinal Function and Structure

To test the hypothesis that EPOR signaling is neuroprotective, it was determined whether hWtEPOR mice with hypoactive EPOR signaling have reduced visual and neural retinal function compared with WT littermates. Visual acuity, assessed by OKT response under both photopic and scotopic conditions at 4, 6, and 8 weeks of age, was not different in hWtEPOR compared with littermate WT mice (Figure 1). Also, a- and b-wave amplitudes under photopic

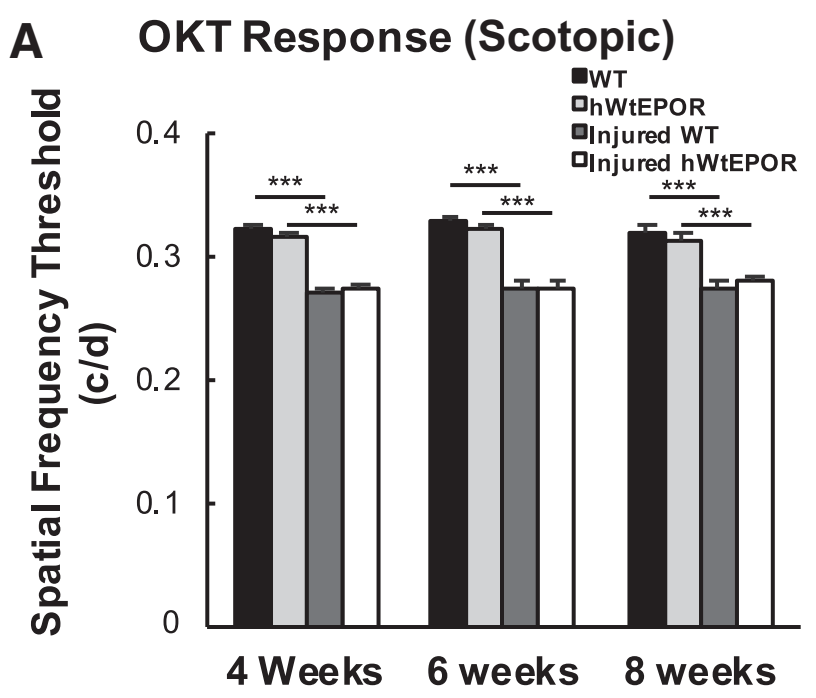

\section{B OKT Response (Photopic)}

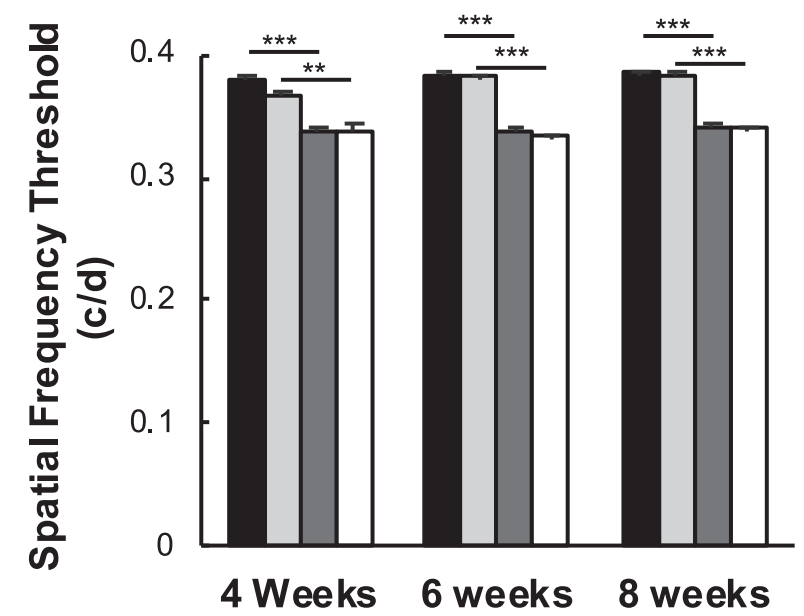

Figure 1 Effect of erythropoietin receptor signaling on visual function. A: Scotopic optomotor kinetic tracking (OKT) response in injured and uninjured WT and hWtEPOR mice at 4, 6, and 8 weeks of age. B: Photopic OKT response in injured and uninjured WT and hWtEPOR mice at 4, 6, and 8 weeks of age. Data are expressed as means $\pm \operatorname{SEM}(\mathbf{A}$ and $\mathbf{B}) . n \geq 8$ eyes per treatment group (A); $n \geq 4$ eyes per treatment group (B). ${ }^{* *} P<0.01$, $* * * P<0.001$. $c /$ d, cycles per degree.

or scotopic conditions and scotopic OP amplitude, measured by Ganzfield ERG, were no different between genotypes at 8 weeks (Figure 2). These findings suggested that hypoactive EPOR signaling did not reduce vision or retinal function. Given that ERG and OptoMotry were not different between the two genotypes, retinal thickness measurements were predicted to be similar (Figure 3). However, the $\mathrm{hWtEPOR}$ mice had thinner central total retinal thickness (Figure 3B), IPL (Figure 3D), and OPL (Figure 3F) compared with littermate control WT mice, suggesting that EPOR signaling was involved in neural retinal structure and potentially connectivity in uninjured mice. These findings suggested that EPOR signaling is important in retinal structure and connectivity in normal conditions but may not 


\section{A A-Wave Amplitude (Scotopic)}

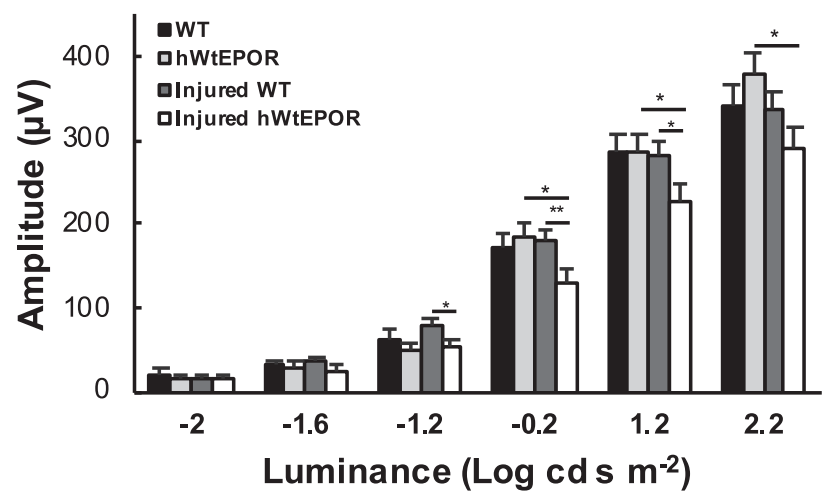

\section{A-Wave Amplitude (Photopic)}

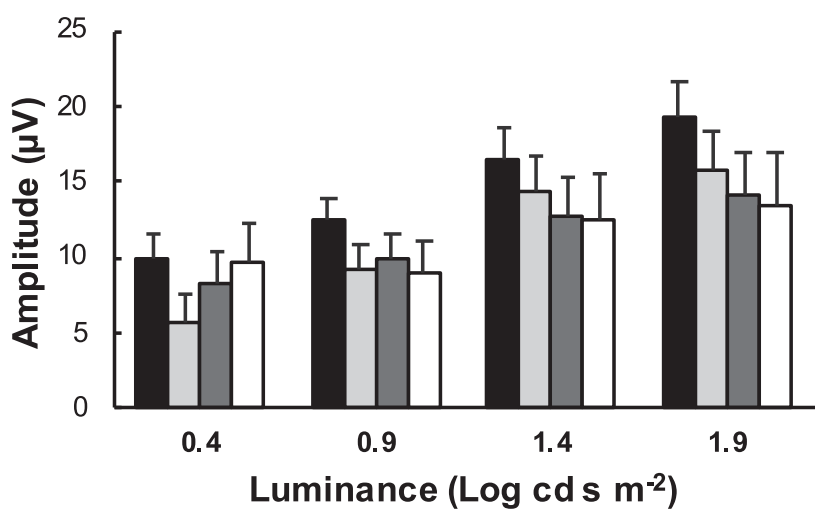

\section{B B-Wave Amplitude (Scotopic)}

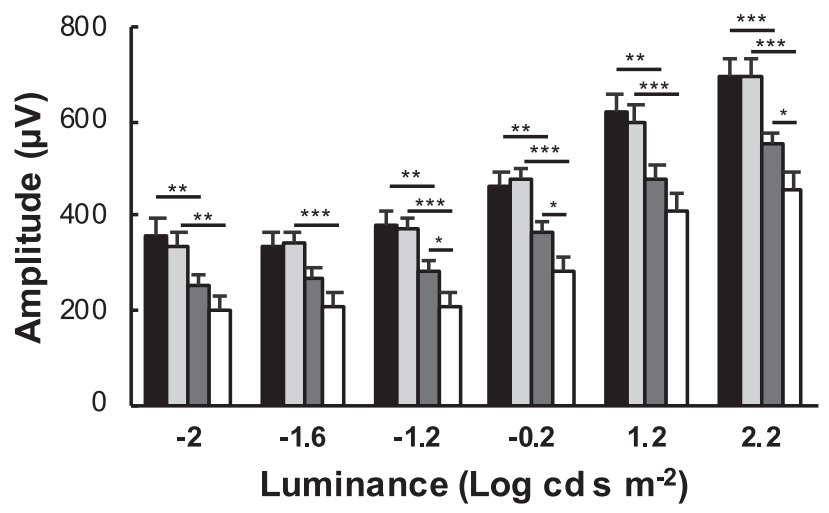

D B-Wave Amplitude (Photopic)

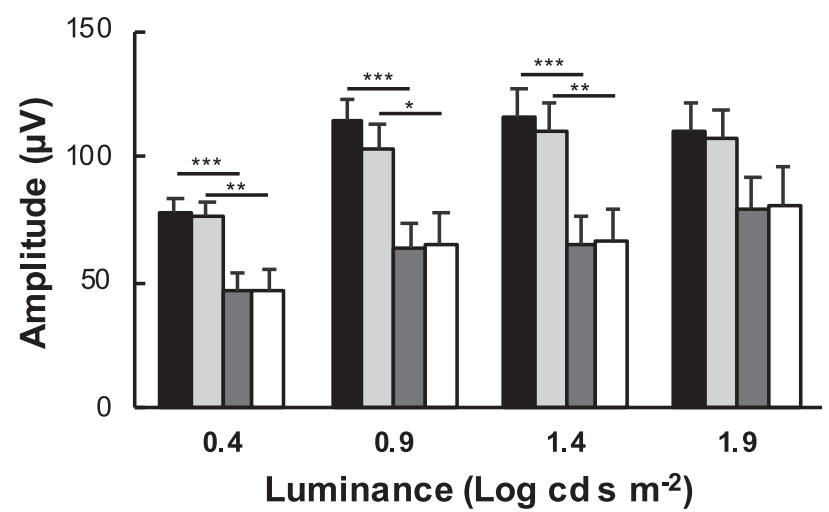

E

OP Amplitude

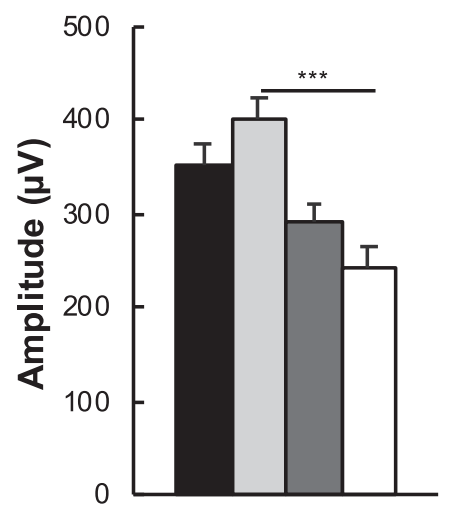

Figure 2 Effect of erythropoietin receptor signaling on electroretinograms. A and B: Scotopic a-wave (A) and b-wave (B) in injured and uninjured WT and hWtEPOR mice at 8 weeks of age. $\mathbf{C}$ and D: Photopic awave (C) and b-wave (D). E: Oscillatory potential (OP) amplitude at maximum scotopic intensity. Hypothesis testing was performed for measurements at the highest intensity; other individual comparisons are provided for descriptive purposes. Data are expressed as means \pm SEM (A-E). $n>7$ mice per treatment group (A and $\mathbf{B}$ ); $n \geq 4$ mice per treatment group (C and $\mathbf{D})$. ${ }^{*} P<0.05$, ${ }^{*} P<<0.01$, and $* * * P<0.001$

have a substantial effect on retinal or visual function (Table 1).

Effect of Reduced EPOR Signaling on Retinal Function after 0xygen-Induced Injury and Repair

The results suggest there is an effect of EPOR signaling on the retina under normal conditions and support the use of the $\mathrm{hWtEPOR}$ mice as a model to assess the effect of EPOR signaling after injury and repair, which translates to aspects of human diseases. To induce a stress common among several retinovascular diseases, the mouse OIR model, ${ }^{21}$ which uses high oxygen to induce central capillary dropout and is followed by regrowth of vessels into the avascular retina when the mice are returned to ambient conditions, was used. Retinal function and structure of mice were analyzed at 8 weeks of age, long after revascularization of the injured retina occurred. ${ }^{16}$ Reduced EPO 

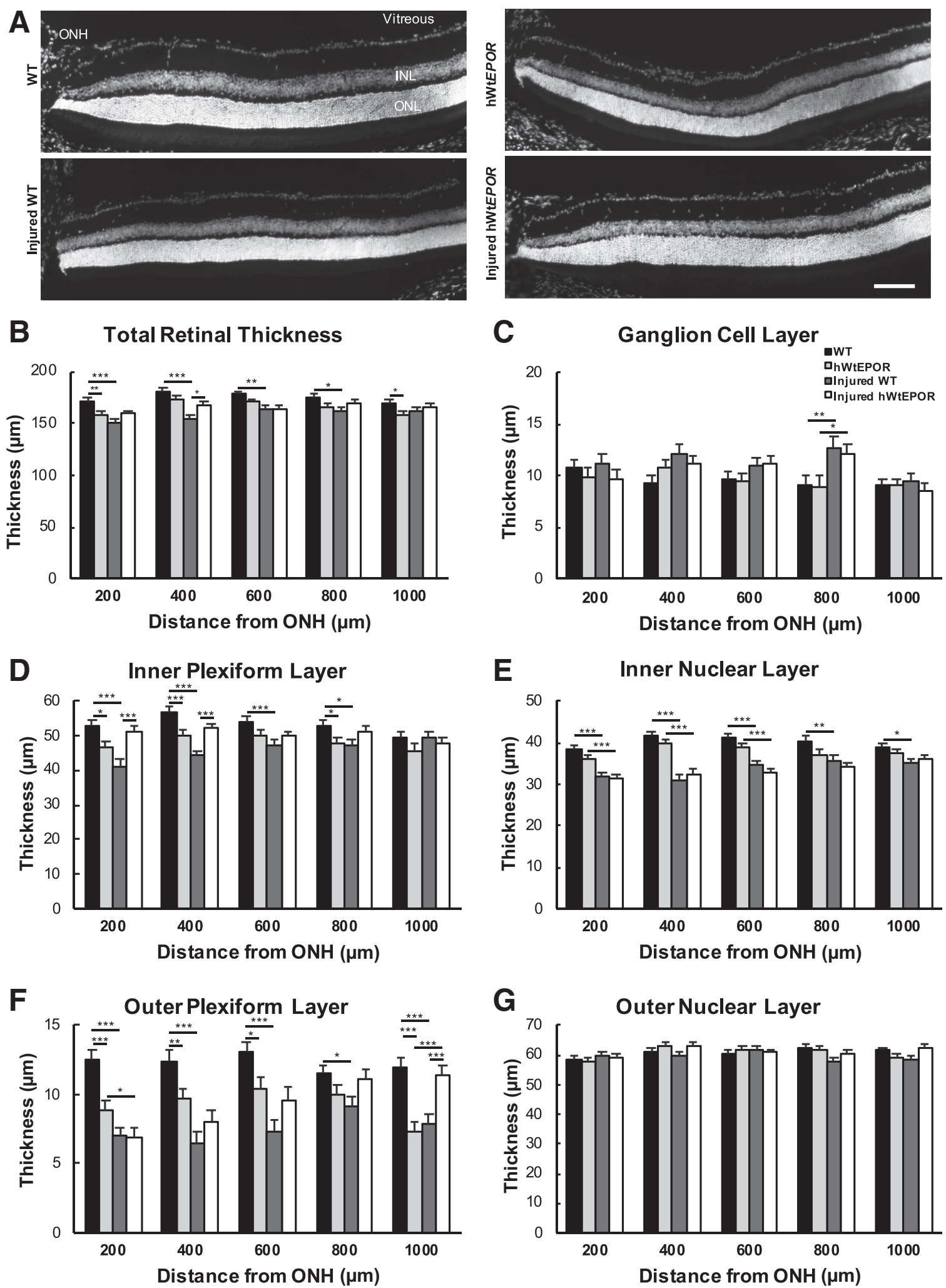

Figure 3 Effect of erythropoietin receptor signaling on retinal structure. A: Representative images of DAPI-stained cryosections from injured and uninjured WT and hWtEPOR mice at 8 weeks of age. B-G: Measurements of total retinal (B), ganglion cell layer (C), inner plexiform layer (D), inner nuclear layer (INL; E), outer plexiform layer $(\mathbf{F})$, and outer nuclear layer $(0 \mathrm{NL} ; \mathbf{G})$ thickness were obtained from each image at 200, 400,600, 800, and 1000 $\mu \mathrm{m}$ from the optic nerve head $(\mathrm{ONH})$. A single eye from at least six mice was cryosectioned for each treatment group; and for each eye, four images containing the 0NH were analyzed. Hypothesis testing was performed for measurements at the central-most and peripheral-most locations (200 and $1000 \mu \mathrm{m}$, respectively); other individual comparisons are provided for descriptive purposes. Data are expressed as means \pm SEM $(\mathbf{B}-\mathbf{G})$. ${ }^{\star} P<0.05, * * P<0.01$, and ${ }^{* * * P<0.001}$. Scale bar $=100$ $\mu \mathrm{m}(\mathbf{A})$. 
Table 1 Key Findings Based on Hypotheses Tested

\begin{tabular}{|c|c|c|c|}
\hline Tested hypothesis & Functional changes & Retinal thickness changes & Cell number changes \\
\hline $\begin{array}{l}\text { Effects of EPOR signaling on } \\
\text { neural retina (uninjured } \\
\text { hWtEPOR mice compared with } \\
\text { WT) }\end{array}$ & $\begin{array}{l}\text { Hypoactive EPOR signaling } \\
\text { resulted in no observed } \\
\text { functional differences }\end{array}$ & $\begin{array}{l}\text { Hypoactive EPOR signaling } \\
\text { resulted in thinner central } \\
\text { retina, thinner central IPL, } \\
\text { and thinner central and } \\
\text { peripheral OPL }\end{array}$ & $\begin{array}{l}\text { Hypoactive EPOR signaling with } \\
\text { increased amacrine cells }\end{array}$ \\
\hline $\begin{array}{l}\text { Effects of injury and repair on } \\
\text { retinal structure and function } \\
\text { (injured WT mice compared } \\
\text { with uninjured WT) }\end{array}$ & $\begin{array}{l}\text { Injury reduced the optomotor } \\
\text { kinetic tracking response } \\
\text { Injury reduced the scotopic } \\
\text { b-wave }\end{array}$ & $\begin{array}{l}\text { Injury resulted in thinner central } \\
\text { retina, thinner central IPL, } \\
\text { and thinner central and } \\
\text { peripheral INL and OPL }\end{array}$ & $\begin{array}{l}\text { Injury decreased central and } \\
\text { peripheral cone numbers } \\
\text { Injury increased RBC ectopic } \\
\text { neurites }\end{array}$ \\
\hline $\begin{array}{l}\text { Effects of EPOR signaling on } \\
\text { injury and repair (injured } \\
\text { hWtEPOR mice compared with } \\
\text { injured WT) }\end{array}$ & $\begin{array}{l}\text { Injury reduced the scotopic } \\
\text { a-wave and oscillatory } \\
\text { potential with hypoactive, but } \\
\text { not normal, EPOR signaling } \\
\text { Injury reduced the scotopic } \\
\text { b-wave in both hypoactive } \\
\text { EPOR and normal EPOR } \\
\text { signaling, but the reduction } \\
\text { was significantly greater with } \\
\text { hypoactive EPOR signaling }\end{array}$ & $\begin{array}{l}\text { Injury resulted in thinner central } \\
\text { retina, thinner central IPL, } \\
\text { and thinner central and } \\
\text { peripheral INL and OPL with } \\
\text { normal EPOR signaling, but } \\
\text { not with hypoactive EPOR } \\
\text { signaling }\end{array}$ & $\begin{array}{l}\text { Injury decreased central and } \\
\text { peripheral cone numbers with } \\
\text { normal, but not hypoactive, } \\
\text { EPOR signaling } \\
\text { Injury decreased peripheral rod } \\
\text { nuclei with hypoactive, but not } \\
\text { normal, EPOR signaling } \\
\text { Injury decreased amacrine cell } \\
\text { numbers with hypoactive, but } \\
\text { not normal, EPOR signaling } \\
\text { Injury increased RBC ectopic } \\
\text { neurites with both normal and } \\
\text { hypoactive EPOR signaling, but } \\
\text { the increase was significantly } \\
\text { greater with normal EPOR } \\
\text { signaling }\end{array}$ \\
\hline
\end{tabular}

EPOR, erythropoietin receptor; INL, inner nuclear layer; IPL, inner plexiform layer; OPL, outer plexiform layer; RBC, rod bipolar cell.

availability in mice after OIR-induced injury was reported to be associated with reduced ERGs compared with mice in OIR with sufficient EPO availability, ${ }^{2}$ but long-term effects of EPOR signaling, as addressed herein, have not been studied.

Induced retinal injury followed by repair led to significantly reduced photopic and scotopic OKT responses at 4, 6 , and 8 weeks of age in both WT and hWtEPOR mice compared with uninjured controls of the same genotype (Figure 1). There was, however, no difference between injured WT and injured hWtEPOR mice. Recovery in visual function of injured mice of either genotype was also not observed from 4 to 8 weeks of age.

Consistent with previous reports, ${ }^{2,30,31}$ there were significantly reduced scotopic b-wave amplitudes of injured compared with uninjured adult WT mice (Figure 2B). However, there was no difference in the scotopic a-wave amplitudes of injured compared with uninjured WT mice (Figure 2A).

Under scotopic conditions, injured hWtEPOR mice had significantly reduced $b$-wave amplitudes compared with either uninjured hWtEPOR or injured WT mice (Figure 2B). Injured hWtEPOR mice also had reduced scotopic a-waves compared with uninjured hWtEPOR mice (Figure 2A). Under photopic conditions, neither injured WT nor injured $\mathrm{hWtEPOR}$ mice had reduced a- or b-wave amplitudes compared with uninjured controls (Figure 2, C and D). The scotopic OP amplitude was significantly lower in injured $\mathrm{hWt} E P O R$ mice compared with uninjured $\mathrm{hWt} E P O R$ mice (Figure 2E), but there was no difference between injured and uninjured WT mice. Taken together, these data suggest that hypoactive EPOR signaling after injury and repair reduces retinal a-wave, b-wave, and OP amplitudes, and the effect is pronounced under scotopic compared with photopic conditions (Table 1).

\section{Effect of Reduced EPOR Signaling on Retinal Structure after 0xygen-Induced Injury and Repair}

Our hypothesis predicted that injured hWtEPOR mice would have more structural and functional change than injured littermate WT mice because of hypoactive EPOR signaling. Compared with uninjured WT mice, injured WT mice had thinner central and peripheral total retinal thicknesses (Figure 3B) and significant thinning of the IPL (Figure 3D) in the central retina, where oxygen-induced damage caused initial capillary loss. However, there was no significant difference in the total retinal thickness of injured hWtEPOR compared with uninjured hWtEPOR mice. Despite the lack of change in hWtEPOR total retinal thickness, hypoactive EPOR signaling affected individual retinal layers after oxygen-induced injury. Although injured 

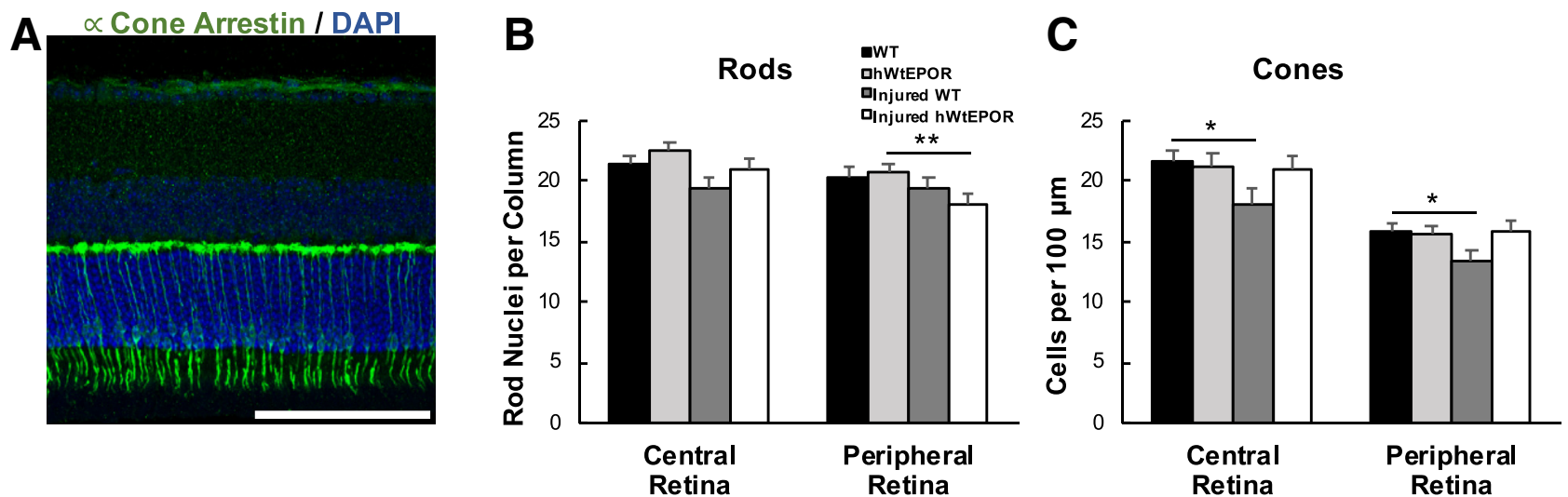

Figure 4 Effect of erythropoietin receptor signaling on rod and cone cells. A: Representative image of a cone arrestin $\alpha-$ and DAPI-stained cryosection from an uninjured WT mouse at 8 weeks of age used to count rod and cones cells. B: Quantification of rod nuclei per column in central and peripheral retina. C: Quantification of cones per $100 \mu \mathrm{m}$ in central and peripheral retina. Data are expressed as means \pm SEM (B and C). $n \geq 8$ images from at least four mice for each treatment group (B and C). ${ }^{*} P<0.05,{ }^{* *} P<0.01$. Scale bar $=100 \mu \mathrm{m}(\mathbf{A})$.

$\mathrm{hWtEPOR}$ mice did not have significantly thinner IPLs compared with uninjured hWtEPOR mice (Figure 3D), the IPLs in the central retina of injured hWtEPOR mice were significantly thicker compared with the central retina of injured WT mice. The OPLs in injured compared with uninjured WT mice were thinner in both central and peripheral retina (Figure 3F), but were only thinned in the central retina of injured compared with uninjured hWtEPOR mice. In the peripheral retina, the OPLs were instead thicker in injured $\mathrm{hWtEPOR}$ compared with injured WT and uninjured $\mathrm{hWtEPOR}$ mice. Both injured WT and hWtEPOR mice had similarly thinned INLs in central retina compared with respective uninjured controls (Figure 3E). There were no significant differences in the ONL based on genotype or injury status at any retinal location measured (Figure 3G).

\section{Effect of Reduced EPOR Signaling on Retinal Cell Numbers}

Injured hWtEPOR mice had significantly reduced a-waves under scotopic conditions (Figure 2A), but no significant thinning of the ONL where rod nuclei are located (Figure $3 \mathrm{G}$ and Table 1). Because thickness measurements do not reflect actual cell number, the number of rod and cone nuclei at both central and peripheral locations from the $\mathrm{ONH}$ were measured in uninjured and injured retinas from
A

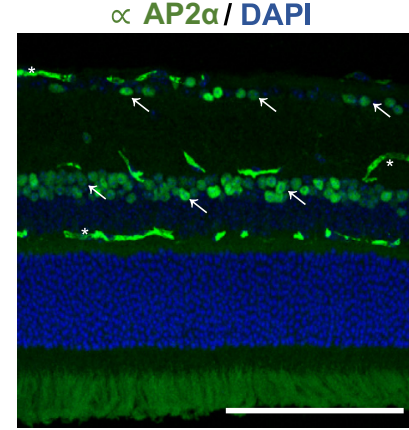

B

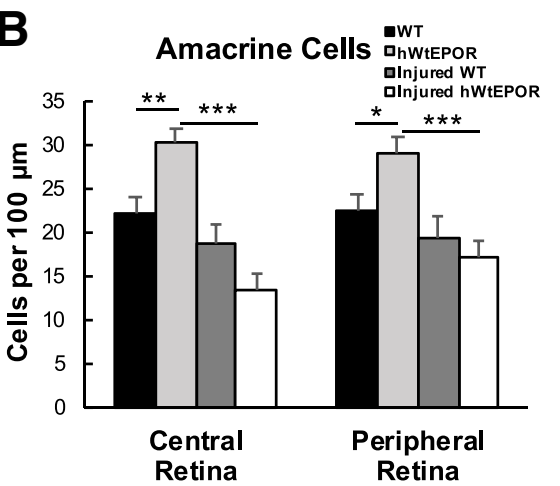

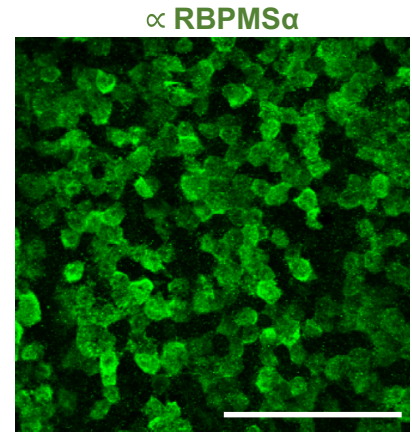

C

Ganglion Cells

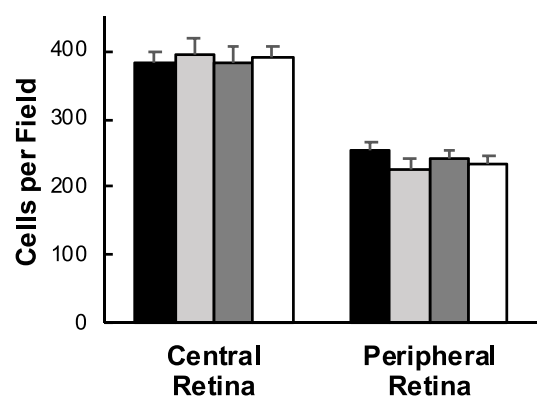

Figure 5 Effect of erythropoietin receptor signaling on amacrine and ganglion cells. A: Representative images of an AP2 $\alpha$ - and DAPIstained cryosection and RBPMS $\alpha$-stained flat mount from an uninjured WT mouse at 8 weeks of age used to count amacrine cells and ganglion cells, respectively. Representative AP $2 \alpha$-positive amacrine cells are noted with arrows. Anti-mouse secondary antibody also labels $\mathrm{Ig}$ in the retinal vasculature, and representative stained vessels are noted with asterisk. B: Quantification of amacrine cells per $100 \mu \mathrm{m}$ in central and peripheral retina. C: Quantification of ganglion cells per field in central and peripheral retina. Data are expressed as means \pm SEM (B and C). $n \geq 8$ images from at least four mice for amacrine cell counts for each treatment group (B); $n \geq 12$ images from at least three mice for ganglion cell counts for each treatment group $(\mathrm{C}) .{ }^{*} P<0.05,{ }^{* *} P<0.01$, and $* * * P<0.001$. Scale bars $=100 \mu \mathrm{m}(\mathbf{A})$. 
mice of both genotypes. There were no significant differences in the number of rod nuclei in the central or peripheral retinas of uninjured WT mice compared with either uninjured hWtEPOR or injured WT mice (Figure 4). However, rod nuclei numbers were reduced in the peripheral retinas of injured compared with uninjured hWtEPOR mice. Cone nuclei numbers were significantly reduced in injured compared with uninjured WT mice in both central and peripheral retinal locations but were unaffected in $\mathrm{hWtEPOR}$ mice (Figure 4). These findings suggest that EPOR signaling was important in maintaining rod nuclei number after injury, whereas cone nuclei number was maintained after injury in mice with hypoactive EPOR signaling.

After injury, hWtEPOR mice had decreased OP amplitude compared with uninjured hWtEPOR mice (Figure 2E). The OP amplitude is believed to, in part, reflect amacrine and ganglion cell function. ${ }^{32,33}$ It was predicted that injured $\mathrm{hWt} E P O R$ mice would have reduced numbers of amacrine and ganglion cells compared with uninjured hWtEPOR mice. Amacrine cell number was significantly higher in uninjured hWtEPOR mice compared with uninjured WT littermates in both the central and peripheral retinal locations (Figure 5). Injured hWtEPOR mice had significantly reduced amacrine cells in both the central and peripheral locations compared with uninjured $\mathrm{hWtEPOR}$ mice, whereas there was no difference in amacrine cell number between uninjured and injured WT mice in central or peripheral retinal locations. Ganglion cell nuclei in flatmounted retinas were not significantly different between genotypes or based on injury status (Figure 5).
Effect of Reduced EPOR Signaling on Rod Bipolar Cell Ectopic Neurites after Injury

The findings of reduced scotopic b-wave amplitudes despite maintained ONL thickness in injured $\mathrm{hWtEPOR}$ compared with injured littermate WT mice led us to question whether rod bipolar cells, which are important in connectivity and in retinal regeneration, were affected by reduced EPOR signaling after injury. The nuclei of rod bipolar cells are normally found in the INL and project neurites into the OPL, but in models of degeneration or regeneration, rod bipolar cells misproject neurites into the ONL and form functional synapses with photoreceptors. ${ }^{34-36}$ Rod bipolar cell number did not significantly differ among treatment groups (Figure 6), and there were no ectopic neurites in either central or peripheral retinas from uninjured mice of either genotype. However, in both injured genotypes, there was a striking increase in the number of ectopic neurites from rod bipolar cell projections into the ONL in the central retina, with a significantly greater number in injured WT than in injured $\mathrm{hWtEPOR}$ mice (Figure 6). There were no ectopic rod bipolar cell neurites in the peripheral retina based on genotype or injury status. These findings suggest that rod bipolar cells are unable to maintain normal connections after injury, potentially accounting in part for reduced b-waves observed in both injured genotypes. This could also in part explain the thinned OPL observed in injured mice. B-waves were significantly lower in injured $\mathrm{hWt} E P O R$ mice than in injured WT mice littermates, suggesting that EPOR signaling is important to limit the
A
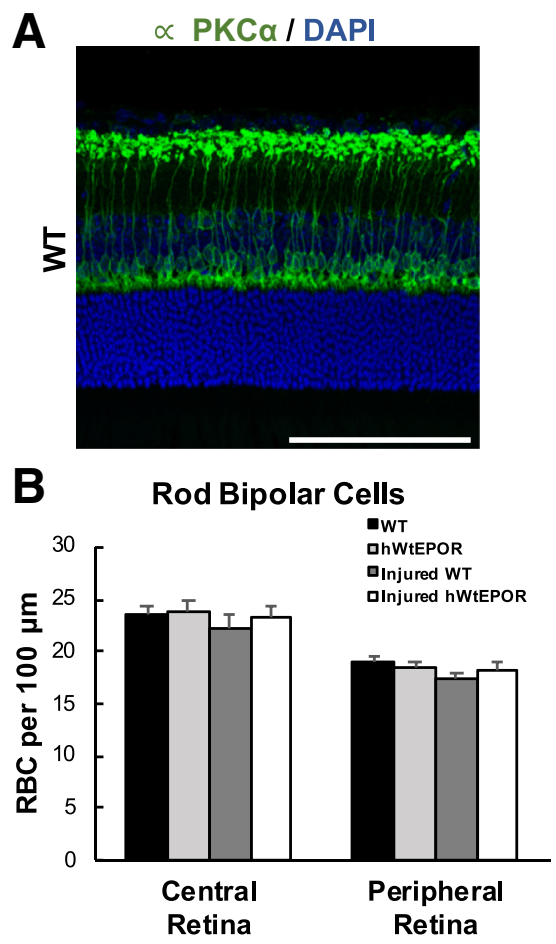
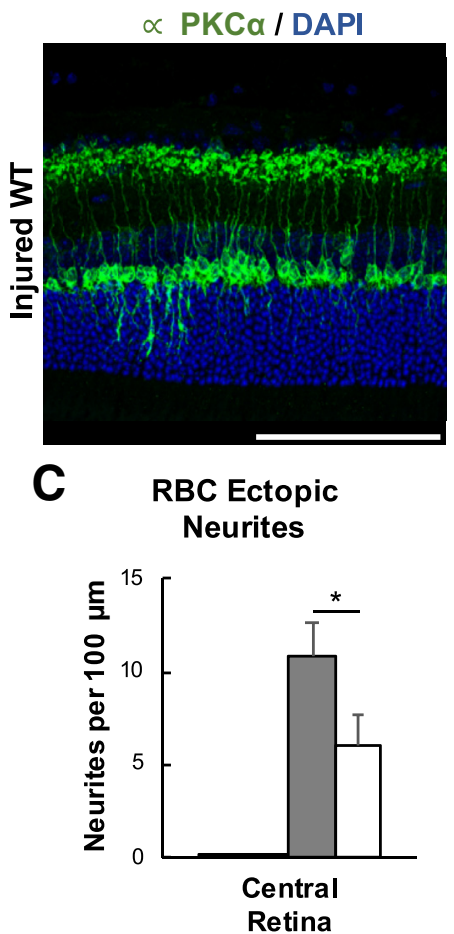

Figure 6 Effect of erythropoietin receptor signaling on rod bipolar cells. A: Representative images of a PKC $\alpha-$ and DAPI-stained cryosection in injured and uninjured WT mice at 8 weeks of age used to count rod bipolar cells and ectopic neurites. B: Quantification of rod bipolar cells per $100 \mu \mathrm{m}$ in central and peripheral retina. C: Quantification of rod bipolar cell ectopic neurites per $100 \mu \mathrm{m}$ in central retina. Data are expressed as means \pm SEM (B and C). $n \geq 8$ images from at least four mice for each treatment group (B and C). ${ }^{*} P<0.05$. Scale bars $=100 \mu \mathrm{m}(\mathbf{A})$. 
reduced neural retinal function from injury through the projection of rod bipolar cell ectopic neurites into the ONL.

\section{Discussion}

Our findings of reduced IPL and OPL and increased amacrine cells in uninjured hWtEPOR mice provide evidence that EPOR signaling is important in neural retinal structure under normal conditions. We propose that in conditions of stress or compromise of the retinal vasculature even with subsequent vascular regrowth, as in the OIR model, retinal bipolar cell ectopic neurites may maintain connectivity to photoreceptors and limit loss of b-wave amplitudes. Furthermore, on the basis of reduced a- and b-wave amplitudes in association with reduced ectopic RBC neurites in injured hWtEPOR compared with injured WT mice, EPOR signaling appears important in limiting the loss of retinal function after injury. These observations may translate to several retinal diseases in which there is initial damage to the retinal vasculature followed by retinal vascular remodeling, such as vascular occlusions related to retinal vein occlusion, inflammation, or potentially diabetic retinopathy.

The murine model of OIR is an example in which a stressor, high oxygen, induces capillary closure of newly developed retinal vessels, followed by regrowth and remodeling of the retinal vasculature. ${ }^{16}$ The injury and regrowth are believed to occur within a few weeks; and although there is some variability in the model, the central retinal areas represent vascularly damaged retina and the peripheral retina represents unaffected retina. Despite the revascularized central retina, there were clear long-term effects of injury, evidenced by reductions in OKT responses, ERG amplitudes, retinal layer thicknesses, and numbers of cells. Our comparison of peripheral retinal findings in injured compared with uninjured adult mice supports the thinking that vascular injury and regrowth directly affects the central retina. However, the observed differences in peripheral OPL thicknesses between injured and uninjured WT and hWtEPOR mice also suggest that other nondirect processes are involved after injury and repair. The finding of increased GCL thickness in injured mice compared with uninjured mice of both genotypes at $800 \mu \mathrm{m}$ despite no difference in ganglion cell numbers between injured and uninjured mice may reflect the transition zone where neovascular budding had acutely occurred at the junction of avascular and vascular retina in the OIR model (Figure 3C).

In previous studies, a single allele knockout of $E P O$ or its receptor has been shown to be lethal. ${ }^{37}$ The humanized knock-in model of hypoactive EPOR signaling reduces EPOR signaling systemically in the mouse without affecting overall survival and provides a comparison between hypoactive and normal EPOR signaling, which may partly translate to the effect of administering systemic EPO. Because the hWtEPOR animals are whole body knock-in mice, it cannot be determined if the effects in this study are due to systemic (indirect) or local (direct) effects of
EPOR signaling. hWtEPOR mice are relatively anemic compared with WT mice having lower hematocrit. ${ }^{9}$ Herein, injury did not significantly impact hematocrit in 8week-old animals (Supplemental Figure S1), but less oxygen delivery may be a factor affecting the retina even after regrowth of vessels.

Previous studies have implicated EPO and EPOR in improved b-wave and OP amplitudes in diabetic experimental models,${ }^{38}$ but other studies have not found an effect of EPOR on maturation or function of retinal cells during aging. ${ }^{39}$ These differences may in part be due to the inherent difficulties of measuring EPOR protein and activation in various tissues with antibodies that have poor specificity, ${ }^{40,41}$ or to the complexity of EPOR signaling and dimerization. ${ }^{14,15,42}$ EPOR has been proposed to dimerize with the $\beta$ common receptor to be tissue protective or with vascular endothelial growth factor receptor 2 to be angiogenic. ${ }^{14,15}$ Human EPOR exhibits decreased stabilization in a transmembrane domain compared with mouse EPOR. ${ }^{19}$ Therefore, hWtEPOR mice have reduced EPOR signaling, but it is unclear whether decreased homodimer stability also impacts EPOR heterodimers. The knock-in mice allow testing the effect of EPOR signaling in vivo independent of EPO levels, because $\mathrm{hWt} E$ POR mice have hypoactive EPOR signaling despite increased EPO levels. ${ }^{18}$ Our studies suggest that even if increased serum $\mathrm{EPO}$ in the hWtEPOR mice triggered signaling through $\beta$ common receptor or vascular endothelial growth factor receptor 2, the effect was not sufficient to protect retinal function after vascular loss and regrowth.

Earlier studies in the mouse model found that EPO stabilized retinal capillaries against high oxygen-induced loss. ${ }^{8}$ These findings cannot be attributed to direct effects of EPOR signaling on neural retina only, and, in fact, our studies find differences between central and peripheral retina, suggesting that central vascular occlusion and regrowth have a substantial effect on the entire retina. Differences were found between hypoactive and normal EPOR signaling, supporting the role of EPOR signaling on events in the peripheral retina after central retinal injury, but these differences also may be due to indirect effects of EPOR on the vasculature and neurons.

Although this study does not address whether supplemental EPO would provide protection in diseases, such as diabetic retinopathy or diabetic retinal neuropathy, it appears to support the hypothesis that at least normal EPOR signaling would reduce retinal damage in diabetic retinopathy. Experimental studies have reported a beneficial effect from intravitreal EPO in models of diabetes. ${ }^{38,43}$ In addition, genetic studies found $E P O$ and $E P O R$ variants associated with risk of diabetic retinopathy, ${ }^{44,45}$ although there is not universal agreement. ${ }^{46}$ A reported feature of diabetic retinopathy is a reduction in total OP amplitude, ${ }^{47,48}$ which was reduced in injured mice with hypoactive EPOR signaling. It is unknown if humans have the same repair or regenerative retinal responses as mice. However, ERG testing is possible in humans and measurement of the effect of EPO on oscillatory potentials may be 
useful in future interventional trials. This study provides evidence the EPOR signaling may be important in reparative processes after injury in the retina.

\section{Author Contributions}

C.A.B. designed and performed the experiments, bred the mice, analyzed the data, and wrote the manuscript; A.B.S. conducted OptoMotry analysis and cryosectioned, stained, and imaged tissue; E.K. conducted electroretinography analyses; A.R., C.K., and I.C. analyzed cell and tissue thickness; M.E.H. designed the experiments, analyzed the data, and wrote the manuscript; M.E.H. is the guarantor of this work and, as such, had full access to all the data in the study and takes responsibility for the integrity of the data and the accuracy of the data analysis.

\section{Supplemental Data}

Supplemental material for this article can be found at http://doi.org/10.1016/j.ajpath.2019.11.009.

\section{References}

1. Shirley Ding SL, Leow SN, Munisvaradass R, Koh EH, Bastion ML, Then KY, Kumar S, Mok PL: Revisiting the role of erythropoietin for treatment of ocular disorders. Eye (Lond) 2016, 30:1293-1309

2. Mowat FM, Gonzalez F, Luhmann UF, Lange CA, Duran Y, Smith AJ, Maxwell PH, Ali RR, Bainbridge JW: Endogenous erythropoietin protects neuroretinal function in ischemic retinopathy. Am J Pathol 2012, 180:1726-1739

3. Grimm C, Wenzel A, Groszer M, Mayser H, Seeliger M, Samardzija M, Bauer C, Gassmann M, Reme CE: HIF-1-induced erythropoietin in the hypoxic retina protects against light-induced retinal degeneration. Nat Med 2002, 8:718-724

4. Diem R, Molnar F, Beisse F, Gross N, Druschler K, Heinrich SP, Joachimsen L, Rauer S, Pielen A, Suhs KW, Linker RA, Huchzermeyer C, Albrecht P, Hassenstein A, Aktas O, Guthoff T, Tonagel F, Kernstock C, Hartmann K, Kumpfel T, Hein K, van Oterendorp C, Grotejohann B, Ihorst G, Maurer J, Muller M, Volkmann M, Wildemann B, Platten M, Wick W, Heesen C, Schiefer U, Wolf S, Lagreze WA: Treatment of optic neuritis with erythropoietin (TONE): a randomised, double-blind, placebocontrolled trial-study protocol. BMJ Open 2016, 6:e010956

5. Wehrle FM, Held U, O'Gorman RT, Disselhoff V, Schnider B, Fauchere JC, Huppi P, Latal B, Hagmann CF: Long-term neuroprotective effect of erythropoietin on executive functions in very preterm children (EpoKids): protocol of a prospective follow-up study. BMJ Open 2018, 8:e22157

6. Juul SE, Mayock DE, Comstock BA, Heagerty PJ: Neuroprotective potential of erythropoietin in neonates; design of a randomized trial Matern Health Neonatol Perinatol 2015, 1:27

7. Entezari M, Flavarjani ZK, Ramezani A, Nikkhah H, Karimi S, Moghadam HF, Daftarian N, Yaseri M: Combination of intravitreal bevacizumab and erythropoietin versus intravitreal bevacizumab alone for refractory diabetic macular edema: a randomized doubleblind clinical trial. Graefes Arch Clin Exp Ophthalmol 2019, 257: $2375-2380$

8. Chen J, Connor KM, Aderman CM, Smith LE: Erythropoietin deficiency decreases vascular stability in mice. J Clin Invest 2008, 118: $526-533$
9. Bretz CA, Divoky V, Prchal J, Kunz E, Simmons AB, Wang H, Hartnett ME: Erythropoietin signaling increases choroidal macrophages and cytokine expression, and exacerbates choroidal neovascularization. Sci Rep 2018, 8:2161

10. Yang Z, Wang H, Jiang Y, Hartnett ME: VEGFA activates erythropoietin receptor and enhances VEGFR2-mediated pathological angiogenesis. Am J Pathol 2014, 184:1230-1239

11. Chen J, Connor KM, Aderman CM, Willett KL, Aspegren OP, Smith LEH: Suppression of retinal neovascularization by erythropoietin siRNA in a mouse model of proliferative retinopathy. Invest Ophthalmol Vis Sci 2009, 50:1329-1335

12. Xiong SQ, Xia XB, Xu HZ, Jiang J: Suppression of retinal neovascularization by small-interference RNA targeting erythropoietin. Ophthalmologica 2009, 223:306-312

13. Morita M, Ohneda O, Yamashita T, Takahashi S, Suzuki N, Nakajima O, Kawauchi S, Ema M, Shibahara S, Udono T, Tomita K, Tamai M, Sogawa K, Yamamoto M, Fujii-Kuriyama Y: HLF/HIF2alpha is a key factor in retinopathy of prematurity in association with erythropoietin. EMBO J 2003, 22:1134-1146

14. Brines M, Cerami A: Discovering erythropoietin's extrahematopoietic functions: biology and clinical promise. Kidney Int 2006, 70:246-250

15. Brines M, Cerami A: The receptor that tames the innate immune response. Mol Med 2012, 18:486-496

16. Lange C, Ehlken C, Stahl A, Martin G, Hansen L, Agostini HT: Kinetics of retinal vaso-obliteration and neovascularisation in the oxygen-induced retinopathy (OIR) mouse model. Graefes Arch Clin Exp Ophthalmol 2009, 247:1205-1211

17. Divoky V, Liu Z, Ryan TM, Prchal JF, Townes TM, Prchal JT: Mouse model of congenital polycythemia: homologous replacement of murine gene by mutant human erythropoietin receptor gene. Proc Natl Acad Sci U S A 2001, 98:986-991

18. Divoky V, Song J, Horvathova M, Kralova B, Votavova H, Prchal JT, Yoon D: Delayed hemoglobin switching and perinatal neocytolysis in mice with gain-of-function erythropoietin receptor. J Mol Med (Berl) 2016, 94:597-608

19. Ebie AZ, Fleming KG: Dimerization of the erythropoietin receptor transmembrane domain in micelles. J Mol Biol 2007, 366:517-524

20. Chang B, Hurd R, Wang J, Nishina P: Survey of common eye diseases in laboratory mouse strains. Invest Ophthalmol Vis Sci 2013, 54:4974-4981

21. Smith LEH, Wesolowski E, McLellan A, Kostyk SK, D’Amato R, Sullivan R, D'Amore PA: Oxygen induced retinopathy in the mouse. Invest Ophthalmol Vis Sci 1994, 35:101-111

22. Redfern WS, Storey S, Tse K, Hussain Q, Maung KP, Valentin JP, Ahmed G, Bigley A, Heathcote D, McKay JS: Evaluation of a convenient method of assessing rodent visual function in safety pharmacology studies: effects of sodium iodate on visual acuity and retinal morphology in albino and pigmented rats and mice. J Pharmacol Toxicol Methods 2011, 63:102-114

23. Thomas BB, Seiler MJ, Sadda SR, Coffey PJ, Aramant RB: Optokinetic test to evaluate visual acuity of each eye independently. J Neurosci Methods 2004, 138:7-13

24. Douglas RM, Alam NM, Silver BD, McGill TJ, Tschetter WW, Prusky GT: Independent visual threshold measurements in the two eyes of freely moving rats and mice using a virtual-reality optokinetic system. Vis Neurosci 2005, 22:677-684

25. Simmons AB, Bloomsburg SJ, Billingslea SA, Merrill MM, Li S, Thomas MW, Fuerst PG: Pou4f2 knock-in Cre mouse: a multifaceted genetic tool for vision researchers. Mol Vis 2016, 22:705-717

26. Haverkamp S, Wassle H: Immunocytochemical analysis of the mouse retina. J Comp Neurol 2000, 424:1-23

27. Rodriguez AR, de Sevilla Muller LP, Brecha NC: The RNA binding protein RBPMS is a selective marker of ganglion cells in the mammalian retina. J Comp Neurol 2014, 522:1411-1443

28. Rueden CT, Schindelin J, Hiner MC, DeZonia BE, Walter AE, Arena ET, Eliceiri KW: ImageJ2: ImageJ for the next generation of scientific image data. BMC Bioinformatics 2017, 18:529 
29. Schindelin J, Arganda-Carreras I, Frise E, Kaynig V, Longair M, Pietzsch T, Preibisch S, Rueden C, Saalfeld S, Schmid B, Tinevez JY, White DJ, Hartenstein V, Eliceiri K, Tomancak P, Cardona A: Fiji: an open-source platform for biological-image analysis. Nat Methods 2012, 9:676-682

30. Villacampa P, Menger KE, Abelleira L, Ribeiro J, Duran Y, Smith AJ, Ali RR, Luhmann UF, Bainbridge JWB: Accelerated oxygen-induced retinopathy is a reliable model of ischemiainduced retinal neovascularization. PLoS One 2017, 12: e0179759

31. Tokunaga CC, Mitton KP, Dailey W, Massoll C, Roumayah K, Guzman E, Tarabishy N, Cheng M, Drenser KA: Effects of anti-VEGF treatment on the recovery of the developing retina following oxygeninduced retinopathy. Invest Ophthalmol Vis Sci 2014, 55:1884-1892

32. Brown KT: The eclectroretinogram: its components and their origins. Vision Res 1968, 8:633-677

33. Wachtmeister L: Oscillatory potentials in the retina: what do they reveal. Prog Retin Eye Res 1998, 17:485-521

34. McGinn TE, Galicia CA, Leoni DC, Partington N, Mitchell DM, Stenkamp DL: Rewiring the regenerated zebrafish retina: reemergence of bipolar neurons and cone-bipolar circuitry following an inner retinal lesion. Front Cell Dev Biol 2019, 7:95

35. Wang $\mathrm{T}$, Pahlberg J, Cafaro J, Frederiksen R, Cooper AJ, Sampath AP, Field GD, Chen J: Activation of rod input in a model of retinal degeneration reverses retinal remodeling and induces formation of functional synapses and recovery of visual signaling in the adult retina. J Neurosci 2019, 39:6798-6810

36. Dorfman AL, Cuenca N, Pinilla I, Chemtob S, Lachapelle P: Immunohistochemical evidence of synaptic retraction, cytoarchitectural remodeling, and cell death in the inner retina of the rat model of oygen-induced retinopathy (OIR). Invest Ophthalmol Vis Sci 2011, 52:1693-1708

37. Wu H, Liu X, Jaenisch R, Lodish HF: Generation of committed erythroid BFU-E and CFU-E progenitors does not require erythropoietin or the erythropoietin receptor. Cell 1995, 83:59-67

38. Liu X, Zhu B, Zou H, Hu D, Gu Q, Liu K, Xu X: Carbamylated erythropoietin mediates retinal neuroprotection in streptozotocin- induced early-stage diabetic rats. Graefes Arch Clin Exp Ophthalmol 2015, 253:1263-1272

39. Caprara C, Britschgi C, Samardzija M, Grimm C: The erythropoietin receptor is not required for the development, function, and aging of rods and cells in the retinal periphery. Mol Vis 2014, 20:307-324

40. Kirkeby A, van Beek J, Nielsen J, Leist M, Helboe L: Functional and immunochemical characterisation of different antibodies against the erythropoietin receptor. J Neurosci Methods 2007, 164:50-58

41. Elliott S, Busse L, Bass MB, Lu H, Sarosi I, Sinclair AM, Spahr C, Um M, Van G, Begley CG: Anti-Epo receptor antibodies do not predict Epo receptor expression. Blood 2006, 107:1892-1895

42. Richmond TD, Chohan M, Barber DL: Turning cells red: signal transduction mediated by erythropoietin. Trends Cell Biol 2005, 15 $146-155$

43. Zhang J, Wu Y, Jin Y, Ji F, Sinclair SH, Luo Y, Xu G, Lu L, Dai W, Yanoff M, Li W, Xu GT: Intravitreal injection of erythropoietin protects both retinal vascular and neuronal cells in early diabetes. Invest Ophthalmol Vis Sci 2008, 49:732-742

44. Abhary S, Burdon KP, Casson RJ, Goggin M, Petrovsky NP, Craig JE: Association between erythropoietin gene polymorphisms and diabetic retinopathy. Arch Ophthalmol 2010, 128:102-106

45. Montesano A, Bonfigli AR, De Luca M, Crocco P, Garagnani P, Marasco E, Pirazzini C, Giuliani C, Romagnoli F, Franceschi C, Passarino G, Testa R, Olivieri F, Rose G: Erythropoietin (EPO) haplotype associated with all-cause mortality in a cohort of Italian patients with type-2 diabetes. Sci Rep 2019, 9:10395

46. Hosseini SM, Boright AP, Sun L, Canty AJ, Bull SB, Klein BE, Klein R, Group DER, Paterson AD: The association of previously reported polymorphisms for microvascular complications in a meta-analysis of diabetic retinopathy. Hum Genet 2015, 134: $247-257$

47. Bresnick GH, Palta M: Oscillatory potential amplitudes: relation to severity of diabetic retinopathy. Arch Ophthalmol 1987, 105: 929-933

48. Tzekov R, Arden GB: The electroretinogram in diabetic retinopathy. Surv Ophthalmol 1999, 44:53-60 